\title{
miR-622 Induces Breast Cancer Cell MCF-7 Proliferation, Migration, and Invasion by Directly Negatively Targeting EYA1
}

\author{
Pan Tang $\mathbb{D}^{1},{ }^{1}$ Yanyan Shen $\mathbb{D}^{1}{ }^{1}$ Jihui Yang, ${ }^{2}$ Nan Wen, ${ }^{1}$ Ying Liu, ${ }^{1}$ Qiqing Zeng, \\ and Tingting Yin ${ }^{1}$ \\ ${ }^{1}$ The Affiliated Nanhua Hospital, Department of Ultrasound Medicine, Hengyang Medical School, University of South China, \\ Hengyang, Hunan 421001, China \\ ${ }^{2}$ Department of Ultrasound Medicine, Hunan Provincial People's Hospital, Changsha, Hunan, China
}

Correspondence should be addressed to Yanyan Shen; syybwk@163.com

Received 5 November 2021; Accepted 13 December 2021; Published 6 January 2022

Academic Editor: Weiguo Li

Copyright (c) 2022 Pan Tang et al. This is an open access article distributed under the Creative Commons Attribution License, which permits unrestricted use, distribution, and reproduction in any medium, provided the original work is properly cited.

\begin{abstract}
Breast cancer is the most common female cancer in the world. Breast cancer patients are currently treated with a combination of surgery, chemotherapy, radiotherapy, and targeted therapy, but the 5-year overall survival rate is still low. Therefore, we plan to explore the potential interaction mechanism between miR-622 and EYA1 in the breast cancer cells and their effect on proliferation, migration, and invasion of breast cancer, to lay a foundation for the gene therapy of breast cancer and improve the therapeutic effect. This study found that miR-622 was highly expressed in breast cancer cell lines, while EYA1 was poorly. In MCF-7 cell line, miR-622 had the highest expression level, while EYA1 had the lowest. Besides, the bioinformatics analysis showed that EYA1 possesses putative miR-622 binding sites within its $3^{\prime} \mathrm{UTR}$. The increased miR-622 significantly enhanced the proliferation, migration, and invasion of MCF-7 cell line and inhibited luciferase reporter activity in the $3^{\prime}$ UTR of EYA1WT. When upregulating the expression of miR-622, the mRNA and protein expression levels of EYA1 were significantly decreased. We also found that the silencing of EYA1 promoted the proliferation, migration, and invasion of breast cancer MCF-7 cell line. These results indicate that miRNA-622 plays a tumor-promoting role in breast cancer through targeted negative regulation of EYA1, suggesting that miRNA-622 may become a potential target for breast cancer treatment.
\end{abstract}

\section{Introduction}

Breast cancer is a malignant tumor growing in the epithelial tissue of the breast. In recent years, the incidence of breast cancer among women in the world has been rising continuously, surpassing lung cancer for the first time and becoming the largest cancer in the world $[1,2]$. It is estimated that by the end of 2020, there will be about 279,100 new confirmed cases of breast cancer in the United States alone [3]. It is the second leading cause of cancer-related deaths among women in the world $[3,4]$. Many studies have made significant progress in early detection, diagnosis, surgery, radiotherapy, and chemotherapy of breast cancer, but there are still a large number of patients who have distant metastasis and become resistant to radiotherapy and chemotherapy [5]. Therefore, it is of great significance to further study the molecular mechanism and signal transduction pathway of breast cancer progression and find new targets for improving breast cancer treatment methods and improving patients' quality of life.

MicroRNAs (miRNAs), small RNA molecules composed of 17-22 nucleotide acids, are able to regulate their target genes directly by binding with their $3^{\prime}$ untranslated regions [6]. It has been estimated that over $30 \%$ of all proteincoding genes in humans are regulated by miRNAs [7]. Due to the difference of miRNA expression between tumor tissues and normal tissues, as well as its characteristics of endogenous, low toxicity, and multitarget, the value of miRNA in tumor diagnosis and treatment has attracted more and more attention. A large number of studies have shown that miRNA plays an important role in the process of breast cancer proliferation and metastasis. For example, 
miR-98-5p regulates breast cancer proliferation by targeting Gab2 [8]. Moreover, miR-19, miR-136, miR-221/222, and other miRNAs have been proved to be related to the invasion and metastasis of breast cancer [9-11].

The miR-622 coding gene is mapped to chromosome 13q31.3. Studies have shown that the expression of miR622 is downregulated in uveal melanoma, human colon cancer, papillary thyroid carcinoma, and other tumors; at the same time, acting as a tumor inhibitor in these types of cancer can inhibit the proliferation, migration, and invasion of a variety of tumor cells from achieving the effect of tumor inhibition by targeting G3BP1, UNC5B, and VEGFA genes [12-14]. Orlandella et al. [15] found that miRNA-622 could inhibit the invasion and migration of breast cancer cell lines MCF-7 and MDA-MB-231 through in vitro studies. Liu et al. [16] also proposed that miR-622 overexpression could inhibit breast cancer cells' epithelial-mesenchymal transition (EMT) by downregulating RNF8, thus inhibiting the migration and invasion of breast cancer cells. However, the exact function and underlying mechanisms of miR-622 in the development of breast cancer need further investigation.

EYA, a member of the retinal determinant gene network (RDGN) family, is a key regulator of eye morphogenesis in Drosophila [17]. Continuing research on the gene has revealed that members of the EYA family have become one of the "tumor markers" because of its overexpression, resistance to cell death, and promotion of angiogenesis, invasion, and metastasis [18]. As a member of its family, EYA1, by binding to SIX1, not only activated STAT3 signaling to promote the development of thyroid cancer but also played a key role in the progression of colorectal cancer $[19,20]$. $\mathrm{Wu}$ et al. [21] studied the expression of EYA1 and found that EYA1 was upregulated in both breast cancer tissues and cell lines. EYA1 promoted the growth of cancer cells by promoting cell proliferation and DNA synthesis and reduced apoptosis. Guan et al. [22] proposed that miR-101 can directly target EYA1 to inhibit the proliferation and induce apoptosis of breast cancer cell [22]. Our bioinformatics analysis showed that EYA1 possesses putative miR-622 binding sites within its $3^{\prime}$ UTR. Therefore, we intend to study the expression of EYA1 and miR-622 in breast cancer cell lines and their interaction mechanism through in vitro experiments to find new targets for breast cancer treatment.

\section{Materials and Methods}

2.1. Cell Culture. Human breast cancer BT474 cell line and the normal human breast epithelial cell line MCF-10A were purchased from Yuchun Biotech, Shanghai, China. MCF-7 and MDA-MB-231 cell lines were acquired from the Clinical Research Institute of the First Affiliated Hospital of University of South China. MCF-7, BT474, and MDA-MB-231 cell lines were cultured in DMEM medium (Gibco, USA) supplemented with $10 \%$ fetal bovine serum (FBS, Gibco, USA) and $1 \%$ penicillin-streptomycin solution (P/S, Gibco, USA). MCF-10A cell line was cultured in the MCF-10A special medium (Yaji Biotech, Shanghai, China, DMEM/F12 (PM150312)+5\%HS (164215-100)+20 ng/mL EGF+0.5 $\mu \mathrm{g} /$ $\mathrm{mL}$ hydrocortisone $+10 \mu \mathrm{g} / \mathrm{mL}$ insulin $+1 \%$ NEAA
$(\mathrm{PB} 180424)+1 \% \mathrm{P} / \mathrm{S}(\mathrm{PB} 180120))$. All cell lines were maintained at $37^{\circ} \mathrm{C}$ in a $5 \% \mathrm{CO}_{2}$ atmosphere. Exponentially growing cell lines were used for the experiments.

\subsection{RNA Extraction and Quantitative Reverse Transcriptase-} Polymerase Chain Reaction ( $q R T-P C R$ ) Analysis. Total RNA was extracted from the cell lines according to the instructions of the RNA extraction kit (Jin Baite Biotech, Beijing, China), and the RNA was reverse-transcribed into cDNA by using the reverse transcription kit (Nuo Weizan Biotech, Nanjing, China). The U6 and GAPDH mRNA were used as internal references. The specific primers of miR-622, EYA1, and U6 were synthesized (Table 1). The qRT-PCR kit (Nuo Weizan Biotech, Nanjing, China) was used for testing. Reaction procedure: predenaturation at $95^{\circ} \mathrm{C} 10 \mathrm{sec} ; 95^{\circ} \mathrm{C} 5 \mathrm{sec}$, $40 \mathrm{x}$; dissolution curve program: $95^{\circ} \mathrm{C} 60 \mathrm{sec} ; 55^{\circ} \mathrm{C} 30 \mathrm{sec}$; $95^{\circ} \mathrm{C} 30 \mathrm{sec}$. After the quantification, the relative quantitative analysis $\left(2^{-\triangle \triangle \mathrm{Ct}}\right)$ was used to measure the relative expression levels of miR-622 and EYA1 mRNA. All the reactions were performed three times.

2.3. Target Prediction. To determine whether EYA1 is a direct target of miR-622, we searched for the potential targets of miR-622 using the prediction programs, TargetScan (http://www.targetscan.org/), miRDB (http://www.mirdb .org/), and microbase (http://www.mirbase.org/).

2.4. Cell Transfection and Small RNA Interference. Take the cells in good condition in the logarithmic growth phase, digest them with pancreatin to make the single-cell suspension, inoculate them into a Petri dish, put them in an incubator overnight, and transfect them when the convergence of cells reaches $30 \%-50 \%$. miR- 622 mimic or its negative control (miR-NC) was transfected into the MCF-7 cells using Ribofactamine transfection reagent (Ruibo Biotech, Guangzhou, China) according to the instructions provided by the manufacturer. After transfection for $6 \mathrm{~h}$, it was changed into a new complete medium and cultured under normal conditions for subsequent experiments. The transfection efficiency was examined according to the green fluorescence detected by a microscope.

To knockdown EYA1 expression, EYA1 siRNA (siEYA1) and its negative control (siRNA-NC) (Ruibo Biotech, Guangzhou, China) were transfected into the MCF-7 cells. Following culture for $48 \mathrm{~h}$, the cells were harvested and the expression level of EYA1 mRNA and protein was measured by qRT-PCR and western blot experiments.

2.5. Western Blot Analysis. Western blot kit (Dingguo Changsheng, Beijing, China) was used to detect the protein expression level of EYA1. Cells in each group were collected, the total protein was extracted with radioimmunoprecipitation assay buffer (RIPA, Yaji Biotech, Shanghai, China), and the protein concentration was detected by BCA Kit (Dingguo Changsheng, Beijing, China). Total protein samples were subjected to electrophoretic separation in an SDS polyacrylamide gel and then were electrotransferred to a PVDF membrane (GE, USA). The membranes were subsequently blocked with blocking fluid (Dingguo Changsheng, Beijing, China). Primary antibodies EYA1 (1:5000, 
TABle 1: Primer sequences for qRT-PCR.

\begin{tabular}{lc}
\hline Name & Sequences \\
\hline Stem loop & GTCGTATCCAGTGCAGGGTCCGAGGTATTCGCACTGGATACGACGCTCCA \\
miR-622 & Forward primers: $5^{\prime}$-GGGTCCGAGGTATTCGCACT- ${ }^{\prime}$ \\
U6 & Reverse primers: $5^{\prime}$-GCACAGTCTGCTGAGGTTGGA-3 ${ }^{\prime}$ \\
EYA1 & Forward primers: $5^{\prime}$-CTCGCTTCGGCAGCACA-3 ${ }^{\prime}$ \\
& Reverse primers: $5^{\prime}$-AACGCTTCACGAATTTGCGT-3 ${ }^{\prime}$ \\
GAPDH & Forward primer: TTTGGCAACTGGTGTACGGG \\
& Reverse primer: GGAGTCGGTCAGGGCTTCA \\
\hline
\end{tabular}

ABclonal, USA, $55 \mathrm{kDa})$ and GAPDH (1:5000, ABclonal, USA, $36 \mathrm{kDa}$ ) were incubated at $4^{\circ} \mathrm{C} 14 \mathrm{~h}$, and then, the membrane was incubated in the secondary antibody (goat anti-rabbit IgG labeled with horseradish peroxidase, $1: 5000$, ABclonal, USA) for $1 \mathrm{~h}$ at $25^{\circ} \mathrm{C}$. The bands were detected by ECL high-efficiency chemiluminescence kit (Genview, USA), and analyzed by ImageJ software. The absorbance of protein bands in each group was determined, and the ratio of target band to GAPDH band was used as the protein expression. The experiment was carried out three times independently.

2.6. CCK-8 Cell Proliferation Assay. According to the manufacturer's instructions, the cell proliferation assay was performed with Cell Counting Kit-8 (CCK-8, Genview, USA). In brief, stably transfected MCF-7 cells were harvested and seeded onto 96-well plates at a density of $5.0 \times 10^{3}$ cells per well, continued to be cultured in a $5 \% \mathrm{CO}_{2}$ incubator at $37^{\circ} \mathrm{C}$. After $12,24,48$, and 72 hours, the cells were treated with $20 \mu \mathrm{L}$ CCK-8 reagent and incubated for $2 \mathrm{~h}$. The OD value at $450 \mathrm{~nm}$ was measured using a microplate imaging system. All experiments were repeated three times.

2.7. Wound Healing Assay. Cell migration was assessed by wound healing assay. After transfection for $48 \mathrm{~h}, \mathrm{MCF}-7$ cells in the logarithmic growth stage were inoculated into 6 -well plates, and the cell concentration was adjusted to 2 $\times 10^{5}$ cells/well. When the cells grew to $80-90 \%$ fusion, a sterile $10 \mu \mathrm{L}$ pipet tip was used to lacerate the center of each hole. After scratching the cells for $0 \mathrm{~h}$, the detached cells were washed with phosphate-buffered saline (PBS), and the remaining cells were cultured at $37^{\circ} \mathrm{C}$ for $12 \mathrm{~h}$. Photographs were taken with the microscope at $0 \mathrm{~h}$ and $12 \mathrm{~h}$. The percentage of wound healing between the two sides of the scratch for each image was measured using Image J software (National Institutes of Health). The cell migration assays were performed three independent times.

2.8. Transwell Assay. MCF-7 cell invasion was assessed in transwell chambers (Becton, Dickinson and Company, USA) following the manufacturer's instructions. Matrigel was coated on the upper chamber of transwell in advance, and then, cells of each group in the logarithmic growth phase after transfection for $48 \mathrm{~h}$ were resuspended in a serum-free medium and inoculated on Matrigel with $1 \times$ $10^{4}$ cells/well. The medium containing 20\% FBS was added into the lower chamber and placed in a $5 \% \mathrm{CO}_{2}$ cell incubator at $37^{\circ} \mathrm{C}$ for $24 \mathrm{~h}$. Cotton swabs wiped the noninvading cells and Matrigel matrix. After fixing with $4 \%$ formaldehyde for $10 \mathrm{~min}$, wash with PBS 3 times, and stain with hematoxylin-eosin (HE, Dingguo Changsheng, Beijing, China). After PBS cleaning, 5 microscopic fields were randomly selected to observe the number of invaded cells (200x). All experiments were duplicated three times.

2.9. Dual-Luciferase Reporter Assay. To construct the wildtype luciferase reporter vector of PGL3-EYA1-WT containing the EYA1 $3^{\prime}$ UTR binding site and the mutant luciferase reporter vector of PGL3-EYA1-MUT without the EYA1 3' UTR binding site, PGL3-EYA1-WT and PGL3-EYA1MUT were cotransfected into cells with miR-622 mimic and miR-NC, respectively. Firefly and Renilla luciferase activities were determined at $24 \mathrm{~h}$ posttransfection by using the Dual-Luciferase Reporter Assay Kit (Thermo Fisher, USA). Calculation formula: relative fluorescence value = firefly luciferase fluorescence value/Renilla luciferase fluorescence value. The assay was carried out three times independently.

2.10. Statistical Analysis. All of the statistical data were analyzed by SPSS 21.0 software. The mean \pm SD presented the results from 3 independent experiments. The $t$-test and one-way analysis of variance was used to determine whether the comparison between two or more groups was statistically significant. $P<0.05$ was considered statistically significant.

\section{Results}

3.1. miR-622 Is Upregulated in Breast Cancer Cell Lines, While EYA1 Is Downregulated. The relative expression level of miR-622 and EYA1 between breast cancer cell lines and normal epithelial cell line was measured by qRT-PCR and western blot. The result showed that miR-622 in BT474, MDA-MB-231, and MCF-7 was upregulated (Figure 1(a)), while EYA1 was downregulated (Figure 1(b)), compared with MCF-10A (miR-622: BT474 vs. MCF-10A, $P=0.010$; MDA-MB-231 vs. MCF-10A, $P=0.001$; MCF-7 vs. MCF10A, $P<0.0001$; EYA1: BT474 vs. MCF-10A, $P=0.028$; 


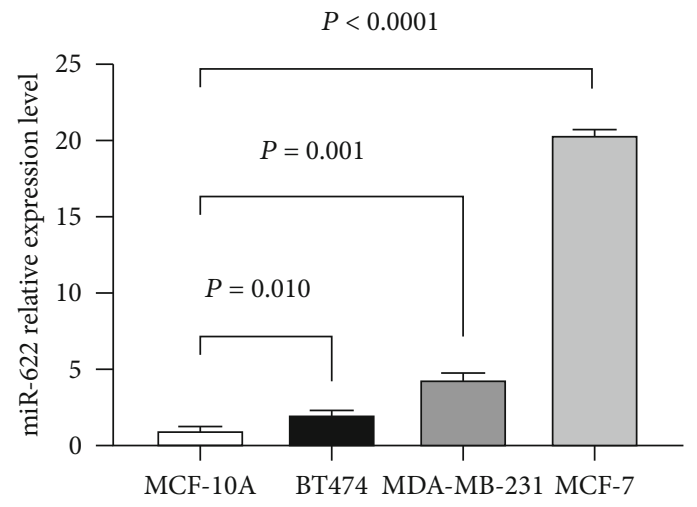

(a)

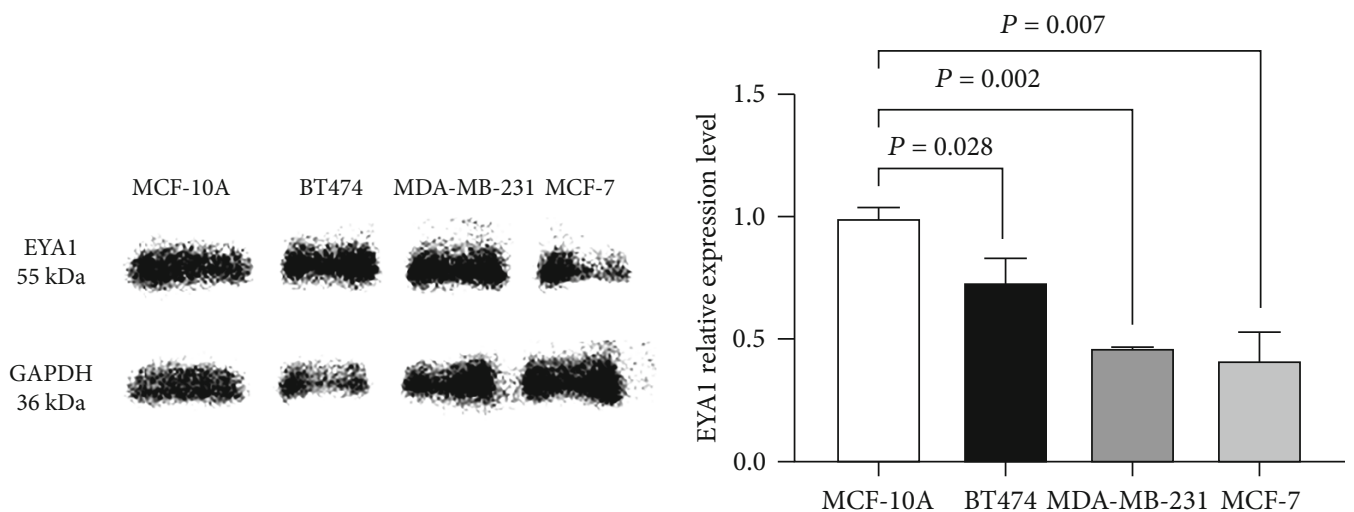

(b)

Figure 1: Expression levels of miR-622 and EYA1 in MCF-10A, BT474, MDA-MB-231, and MCF-7 cell lines. (a) The expression of miR622 was increased in three breast cancer cell lines (BT474, MDA-MB-231, and MCF-7) compared to breast normal epithelial cell line MCF$10 \mathrm{~A}$, detected by using qRT-PCR. The expression level was the highest in MCF-7 (BT474 vs. MCF-10A, $P=0.010$; MDA-MB-231 vs. MCF$10 \mathrm{~A}, P=0.001$; MCF-7 vs. MCF-10A, $P<0.0001$ ). (b) The expression of EYA1 was decreased in three breast cancer cell lines (BT474, MDAMB-231, and MCF-7) compared to breast normal epithelial cell line MCF-10A, detected by using qRT-PCR and western blot. The expression level was the lowest in MCF-7 (EYA1: BT474 vs. MCF-10A, $P=0.028$; MDA-MB-231 vs. MCF-10A, $P=0.002$; MCF-7 vs. MCF-10A, $P=0.007$ ).

MDA-MB-231 vs. MCF-10A, $P=0.002 ; \mathrm{MCF}-7$ vs. MCF$10 \mathrm{~A}, P=0.007)$. MCF-7 cell line was selected for the following experiment due to the expressions of miRNA and EYA1, in which miR-622 was much higher while EYA1 was lower than that of other breast cancer cell lines.

3.2. Overexpression of miR-622 Promotes Breast Cancer Cell Proliferation, Migration, and Invasion. In order to investigate the role of miR-622 in breast cancer, an experiment in vitro was conducted. miR-622 mimic or miR-NC was transfected into MCF-7 cell line, respectively, for further culture. The result showed that the expression of miR-622 was promoted by mimic compared with the miR-NC or control group after the transfection for $48 \mathrm{~h}$ (Figure 2(a)) (miR-622 mimic vs. miR-NC, $P=0.007$; miR-622 mimic vs. control group, $P=0.011)$. Furthermore, CCK- 8 assay, wound healing assay, and transwell chamber assay was conducted to reveal the roles of miR-622 in proliferation, migration, and invasion of MCF-7 cells. We found that the overexpression of miR-622 significantly promoted cell proliferation, migration, and invasion compared with the miR-NC or control group (Figures 2(b)-2(f)) (proliferation: miR-622 mimic vs. miR-NC, $P=0.009$; miR-622 mimic vs. control group, $P=0.030$; migration: miR-622 mimic vs. miR-NC, $P=$ 0.023; miR-622 mimic vs. control group, $P=0.011$; invasion: miR-622 mimic vs. miR-NC, $P=0.033$; miR-622 mimic vs. control group, $P=0.032$ ), which indicates that miR-622 might serve as a tumor promoter in regulating breast cancer biological process.

3.3. EYA1 Was the Direct Target of miR-622 in Breast Cancer. Here, we explored the molecular mechanisms of the tumor-promoting effect of miR-622 on breast cancer. Firstly, through TargetScan (http://www.targetscan.org/), miRDB (http://www.mirdb.org/), and microbase (http:// www.mirbase.org/) software database screening, we found that EYA1 was a predicted target of miR-622 and that there were two binding sites of miR-622 in the $3^{\prime} \mathrm{UTR}$ of EYA1 (Figures 3(a) and 3(b)). According to the context++ score and the context++ score percentile, position 1779-1786 was selected for further analysis, suggesting that EYA1 may be a potential target of miR-622. Further luciferase reporter assay proved miR-622 could directly target EYA1. When we cotransfected the reporter vectors containing wild-type 


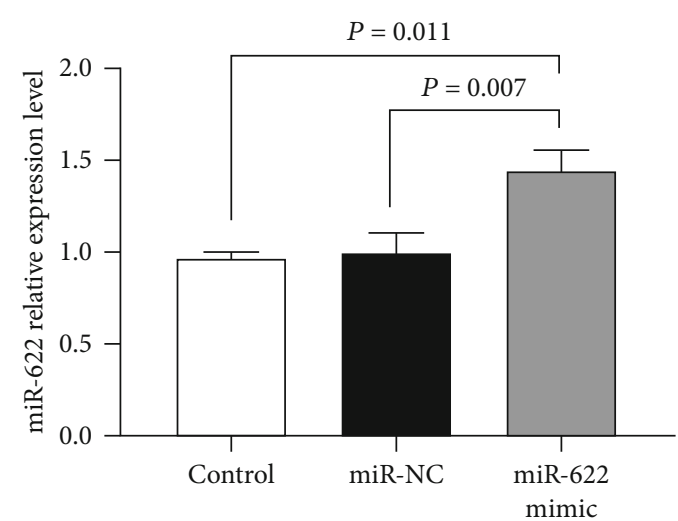

(a)
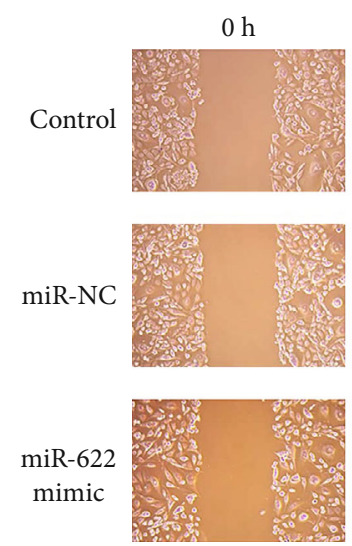

(c)

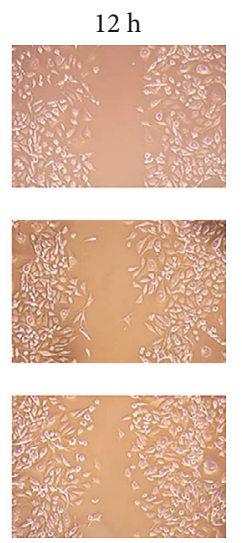

miR-62

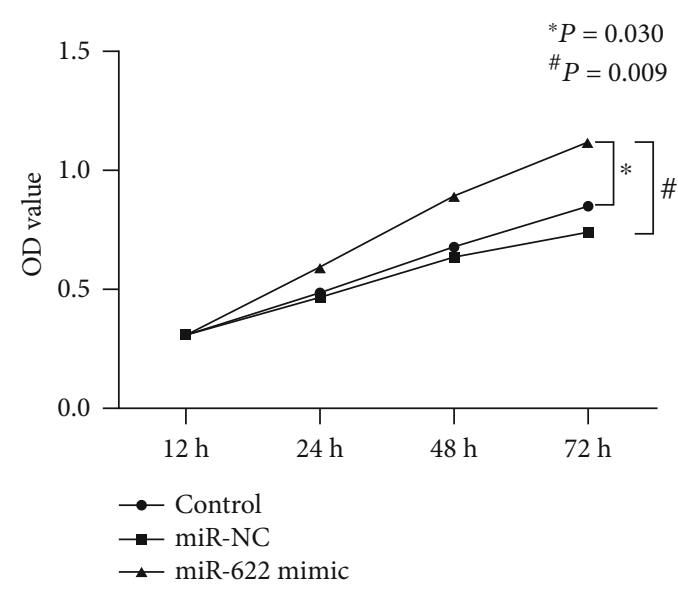

(b)

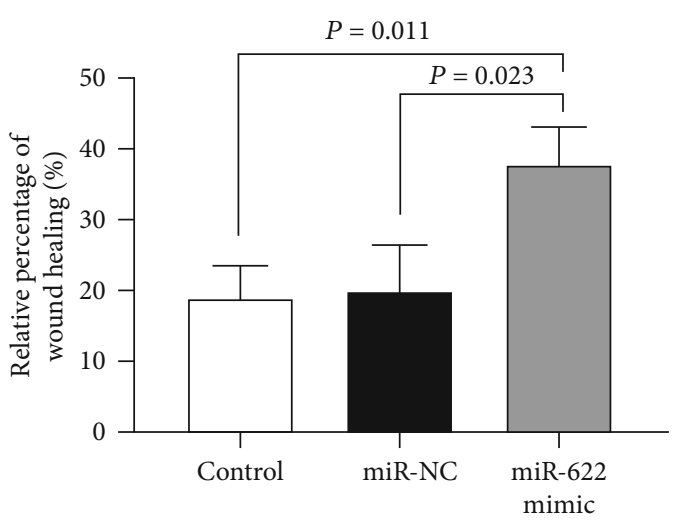

(d)

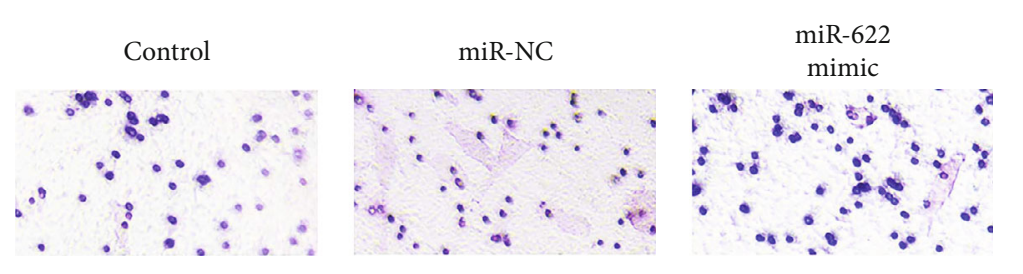

(e)

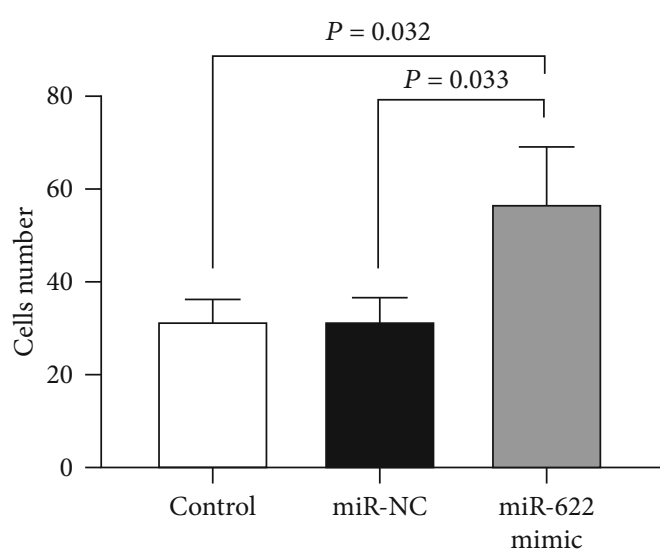

(f)

FIGURE 2: Overexpression of miR-622 could promote breast cancer cell proliferation, migration, and invasion. (a) After transfection of miR622 mimic, the relative expression level of miR-622 in the miR-622 mimic group was higher than that in the miR-NC and control group (miR-622 mimic vs. miR-NC, $P=0.007$; miR-622 mimic vs. control group, $P=0.011$ ). (b) After transfection of miR-622 mimic for 24 hours, the proliferation of the miR-622 mimic group was stronger than that of the miR-NC and control group (miR-622 mimic vs. miRNC, $P=0.009$; miR-622 mimic vs. control group, $P=0.030)$. (c, d) The mobility in the miR-622 mimic group $(37.79 \% \pm 5.42 \%)$ was higher than that in the miR-NC group $(19.96 \% \pm 6.53 \%)$ and the control group $(18.95 \% \pm 4.53 \%)(\mathrm{miR}-622 \mathrm{mimic}$ vs. miR-NC, $P=$ 0.023 ; miR-622 mimic vs. control group, $P=0.011)$. (e,f) The number of invasive cells in the miR-NC and control group was lower than that in the miR-622 mimic group (miR-622 mimic vs. miR-NC, $P=0.033$; miR-622 mimic vs. control group, $P=0.032$ ).

EYA1 3'UTR (PGL3-EYA1-WT) and miR-622 mimics into MCF-7 cells, luciferase activity was significantly reduced (miR-622 mimic vs. miR-NC, $P=0.002$ ). On the contrary, when we cotransfected the reporter vectors containing mutant EYA1 3'UTR (PGL3-EYA1-MT) and miR-622 mimics, luciferase activity was not changed (Figure 3(c)) 


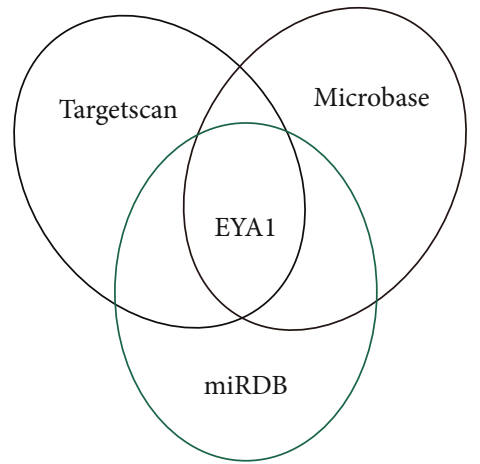

(a)

\begin{tabular}{|c|c|c|c|c|}
\hline & $\begin{array}{l}\text { Predicted consequential pairing of target region (top) and } \\
\text { miRNA (bottom) }\end{array}$ & $\begin{array}{l}\text { Site } \\
\text { type }\end{array}$ & $\begin{array}{l}\text { Context++ } \\
\text { score }\end{array}$ & $\begin{array}{l}\text { Context++ score } \\
\text { percentile }\end{array}$ \\
\hline $\begin{array}{l}\text { Position 376-382 of EYA1 3' UTR } \\
\text { hsa-miR-622 }\end{array}$ & $\begin{array}{c}\text {...CUAAUUGCACAACAGAGACUGAA... } \\
\text { CGAGGUUGGAGUCGUCUGACA }\end{array}$ & $\begin{array}{l}7 \text { mer- } \\
\text { A1 }\end{array}$ & -0.14 & 84 \\
\hline $\begin{array}{l}\text { Position 1779-1786 of EYA1 3' UTR } \\
\text { hsa-miR-622 }\end{array}$ & $\begin{array}{c}5^{\prime} \text {...UGUUUUUGGACUCUGCAGACUGA... } \\
3^{\prime} \text { CGAGGUUGGAGUCGUCUGACA }\end{array}$ & 8 mer & 0.32 & 97 \\
\hline
\end{tabular}

(b)

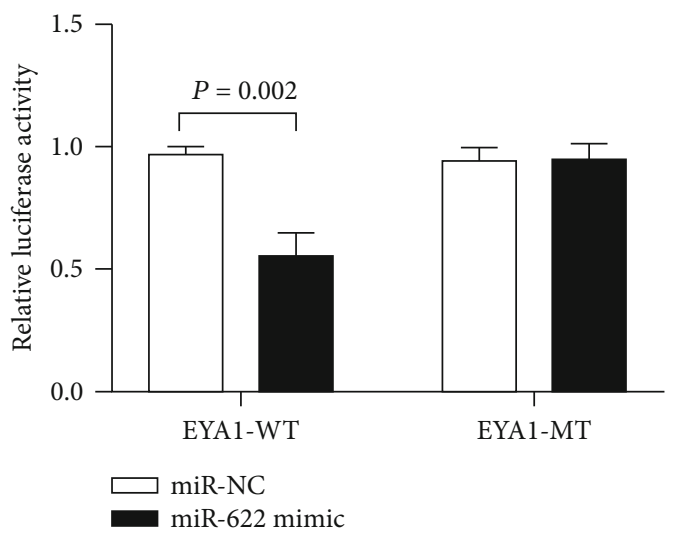

(c)

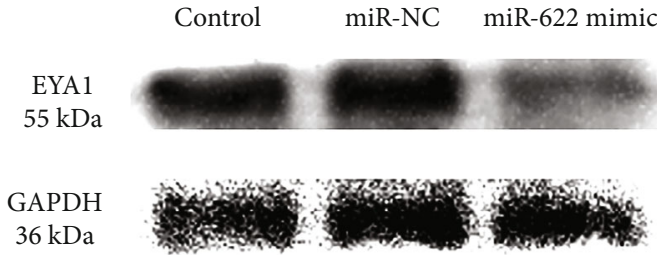

(e)

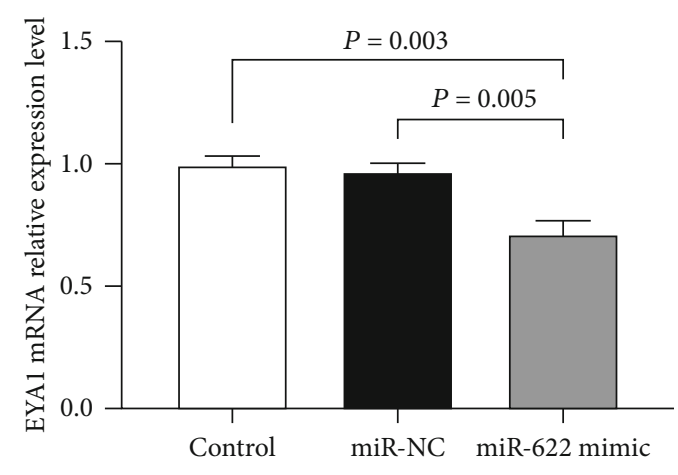

(d)

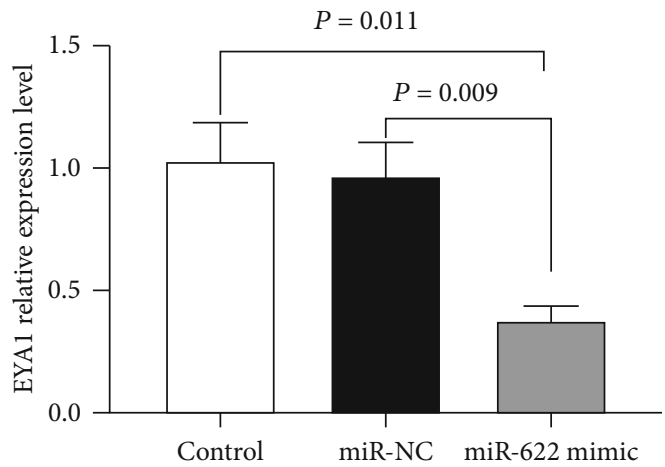

(f)

FIgURE 3: EYA1 was the direct target of miR-622 in breast cancer. (a, b) through TargetScan, miRDB, and microbase software database screening, we found that EYA1 was a predicted target of miR-622 and that there were two binding sites of miR-622 in the $3^{\prime} \mathrm{UTR}$ of EYA1. (c) miR-622 only affects the wild-type $3^{\prime}$ UTR of EYA1, but not the mutant-type, as determined by the luciferase assay (EYA1WT: miR-622 mimic vs. miR-NC, $P=0.002$; EYA1-MT: miR-622 mimic vs. miR-NC, $P>0.05$ ). (d-f) After transfection of miR-622 mimic, the relative expression levels of EYA1 mRNA and protein in the miR-622 mimic group were lower than those in the miR-NC and control group (EYA1 mRNA: miR-622 mimic vs. miR-NC, $P=0.005$; miR-622 mimic vs. control group, $P=0.003$; EYA1 protein: miR-622 mimic vs. miR-NC, $P=0.009$; miR-622 mimic vs. control group, $P=0.011$ ). 
(miR-622 mimic vs. miR-NC, $P>0.05$ ). In addition, after transfection for 48 hours, we found that the overexpression of miR-622 significantly decreased the expression of EYA1 mRNA and protein compared with miR-NC or the control group (Figures 3(d)-3(f)) (EYA1 mRNA: miR-622 mimic vs. miR-NC, $P=0.005$; miR-622 mimic vs. control group, $P=0.003$; EYA1 protein: miR-622 mimic vs. miR-NC, $P=$ 0.009; miR-622 mimic vs. control group, $P=0.011$ ).

3.4. Roles of EYA1 in Proliferation, Migration, and Invasion of Breast Cancer Cells. In order to further investigate the role of EYA1 in breast cancer, an experiment in vitro was conducted. si-EYA1 or siRNA-NC was transfected into MCF-7 cell line, respectively, for further culture. The result showed that si-EYA1 inhibited the expression of EYA1 mRNA and protein expression compared with the siRNA-NC or control group (Figures 4(a)-4(c)) (EYA1 mRNA: si-EYA1 vs. siRNA-NC, $P=0.011$; si-EYA1 vs. control group, $P=0.012$ ; EYA1 protein: si-EYA1 vs. siRNA-NC, $P=0.033$; siEYA1 vs. control group, $P=0.004)$. Furthermore, CCK-8 assay, wound healing assay, and transwell chamber assay were conducted to reveal the roles of EYA1 in proliferation, migration, and invasion of MCF-7 cells. The results showed that the silencing of EYA1 significantly promoted cell proliferation, migration, and invasion compared with the siRNANC or control group (Figures 4(d)-4(h)) (proliferation: siEYA1 vs. siRNA-NC, $P=0.004$; si-EYA1 vs. control group, $P=0.001$; migration: si-EYA1 vs. siRNA-NC, $P=0.046$; siEYA1 vs. control group, $P=0.049$; invasion: si-EYA1 vs. siRNA-NC, $P=0.003$; si-EYA1 vs. control group, $P=0.003$ ).

\section{Discussion}

Breast cancer is the most common cancer among women. According to the latest global cancer data in 2020, breast cancer has replaced lung cancer as the most common cancer in the world [1]. Distant organ metastases account for $90 \%$ of breast cancer deaths [23]. Therefore, there is an urgent need to understand the biological mechanism of breast cancer and find new therapeutic targets to improve the disease treatment and patients' survival rates.

Molecular targeted therapy is a systemic treatment that can effectively improve disease-free survival in operable breast cancer [24]. Since its discovery in 1993, more and more evidence had shown that various miRNAs' abnormal expression is involved in the occurrence and development of various tumors. Downregulated or upregulated miRNAs are advocated as new prognostic biomarkers and therapeutic targets. For example, in metastatic breast cancer, the expression levels of miR-21 and miR-105 were significantly higher than those in healthy subjects $[25,26]$. miR-21 was significantly associated with the advanced clinical stage, the metastasis of the cancer cells in a lymph node, and poor prognosis [27]. Therefore, the study of the biological role of miRNAs in cancer opens up a new approach for cancer diagnosis and treatment.

miR-622, a member of the miRNA family, has been presented in various cancers as an oncogene or tumor suppressor gene since its discovery in 2010 [28]. Our results showed that the expression level of miR-622 in breast cancer cell lines was higher than that in normal human breast epithelial cell line, and the expression level of miRNA-622 was the highest in MCF-7 cell line. In order to understand the effect of miR-622 on the proliferation, migration, and invasion of breast cancer, we transfected breast cancer MCF-7 cell line with miR-622 mimic and found that the proliferation, migration, and invasion ability of MCF-7 cell line was significantly enhanced, when miR-622 was overexpressed. Therefore, overexpression of miR-622 significantly promoted the proliferation, migration, and invasion of MCF-7 cells. These results suggest that miR-622 may play a promoting role in breast cancer progression. However, related studies have shown that in various cancers, including breast cancer, miR-622, as a tumor suppressor gene, can directly act on the corresponding targets to inhibit tumor proliferation, migration, and invasion, respectively. miR-622 can directly target CCL18/MAPK, c-myc, and E2F1 to inhibit the progression of renal carcinoma, cholangiocarcinoma, and human esophageal squamous cell carcinoma, separately [29-31]. Furthermore, miR-622 has been shown to target RNF8 and NUAK1 kinase in breast cancer to inhibit its progression [15, 16]. In addition, Orlandella et al. [15], in the study of patients with ductal breast carcinoma, found that miR-622 was low expressed in the plasma of these patients, and miR-622 expression was negatively correlated with advanced tumor grade and aggressive clinicopathological features with high levels of Ki67. These conclusions are contrary to our findings that miR-622 is a procancer factor. However, the bioinformatics identification of Liu et al. [16] supports our study, showing that high levels of miR-622 expression were inversely associated with patient survival in all breast cancer patients in TCGA data set. Therefore, miR-622 can act as both a tumor suppressor gene and an oncogene in breast cancer, which may be associated with the high heterogeneity of breast cancer or different tumor sources and cell line classifications. Similarly, miR-622 can be used as both oncogene and tumor suppressor gene in colorectal cancer. Wang et al. [32] reported that miR-622 was upregulated in colorectal cancer tissues and cells, and it was used as a promoter in colorectal cancer. When miR622 expression was decreased, the migration and invasion of colorectal cancer cells were inhibited. However, Fang et al.'s [33] research found that miR-622 was downregulated in colorectal cancer tissues and cells; the increased miR-622 inhibited CRC cell proliferation and migration, while inhibition of miR-622 reversed this phenomenon in CRC cells. In addition, Liu et al. [16] pointed out that due to the difference in the tumor microenvironment, whether miR-622 is carcinogenic or anticancer cannot be generalized [16].

Through bioinformatics analysis, we found that the $3^{\prime}$ UTR of EYA1 has putative binding sites of miRNA-622. Therefore, to explore the relationship between miR-622 and EYA1 and find out the related mechanism of miR-622 promoting breast cancer cell lines, we launched a study on EYA1.

As one of the four mammalian EYA homologous genes, EYA1 has attracted extensive attention in the role of oncogenesis and development in recent years. Studies have shown that EYA1 is overexpressed as a tumor promoter in various types of cancer, including breast cancer, glioblastoma, and 


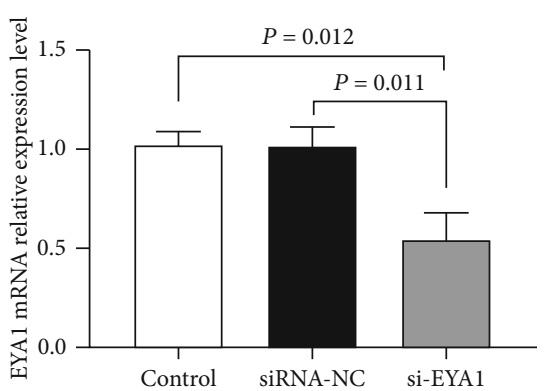

(a)

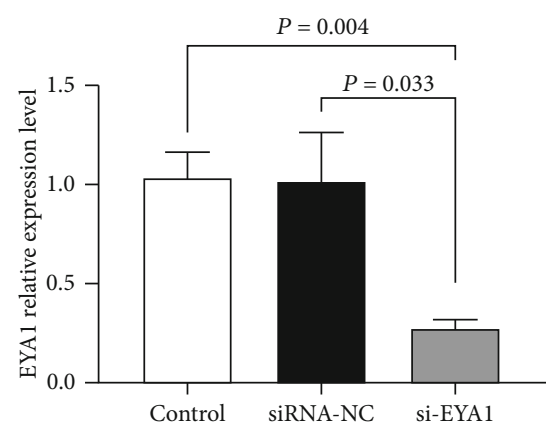

(c)

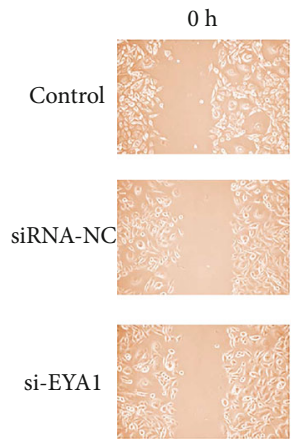

(e)

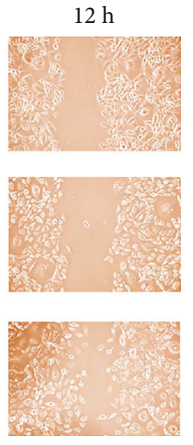

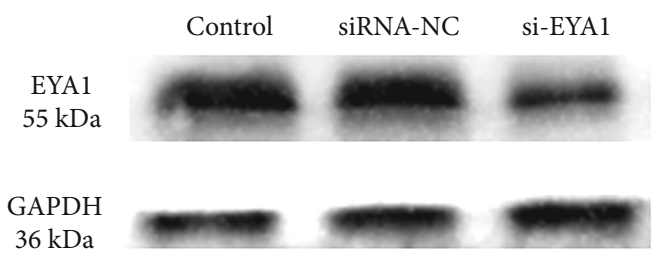

(b)

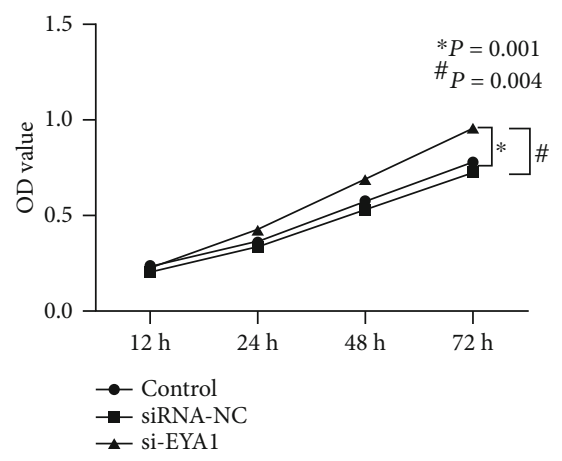

(d)

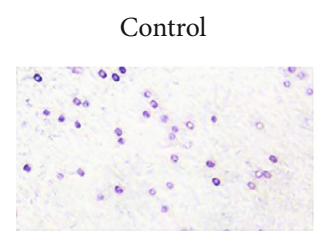

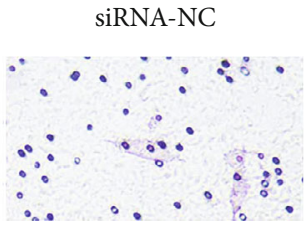

(g)

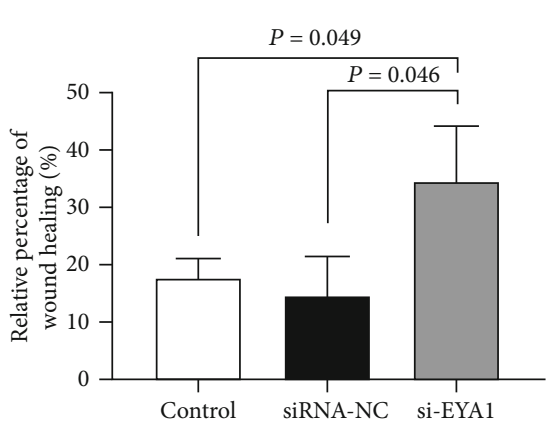

(f)
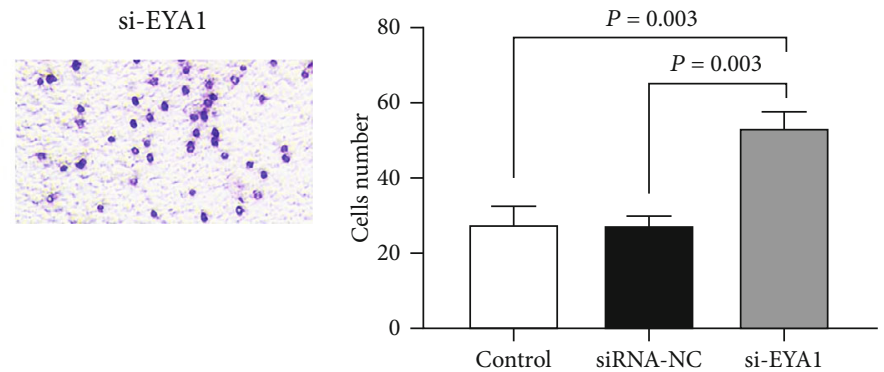

(h)

FIGURE 4: Roles of EYA1 in proliferation, migration, and invasion of breast cancer cells. (a-c) After transfection of si-EYA1, the relative expression levels of EYA1 mRNA and protein in the si-EYA1 group were lower than those in the siRNA-NC and control group (EYA1 mRNA: si-EYA1 vs. siRNA-NC, $P=0.011$; si-EYA1 vs. control group, $P=0.012$; EYA1 protein: si-EYA1 vs. siRNA-NC, $P=0.033$; si-EYA1 vs. control group, $P=0.004$ ). (d) After transfection of si-EYA1 for 48 hours, the proliferation of the si-EYA1 group was stronger than that of the siRNA-NC and control group (si-EYA1 vs. siRNA-NC, $P=0.004$; si-EYA1 vs. control group, $P=0.001$ ). (e, f) The mobility in the si-EYA1 group $(34.40 \% \pm 9.81 \%)$ was higher than that in the siRNA-NC group $(14.60 \% \pm 7.93 \%)$ and the control group $(17.63 \% \pm 3.52 \%)$ (si-EYA1 vs. siRNA-NC, $P=0.046$; si-EYA1 vs. control group, $P=0.049)$. $(\mathrm{g}, \mathrm{h})$ The number of invasive cells in the control group and the siRNA-NC group was lower than that in the si-EYA1 group (si-EYA1 vs. siRNA-NC, $P=0.003$; si-EYA1 vs. control group, $P=0.003)$. 
hepatocellular carcinoma [22, 34-36]. In addition, in breast cancer, related studies indicate that breast cancer with high levels of EYA1 has a poor prognosis, and EYA1 has been independently identified as a prognostic biomarker for breast cancer. Exogenous expression of EYA1 promoted the growth of breast tumors and induced the properties of tumor stem cells (CSCs) by activating cyclin D1 [21]. However, our results showed that the expression level of EYA1 in breast cancer cell lines was lower than that in normal human breast epithelial cell line, and the expression level of EYA1 was the lowest in MCF-7 cell line with the highest miRNA-622 expression level. Therefore, we chose MCF-7 cell line for subsequent experiments. In order to confirm the relationship between miR-622 and EYA1, we conducted a dual-luciferase reporter gene assay. The results showed that increased miR-622 level inhibited the activity of luciferase reporter in the $3^{\prime}$ UTR of EYA1-WT. The overexpression of miRNA-622 inhibited EYA1 mRNA and protein expression level, which confirmed that EYA1 was a direct target of miRNA-622. At the same time, we silenced the expression of EYA1 gene and the result showed that the decreased expression of EYA1 had the same effect as miRNA622, which is, in MCF-7 cells, overexpression of miRNA-622 or silencing of EYA1 significantly enhanced the proliferation, migration, and invasion of breast cancer cells. Therefore, we speculated that miR-622 might play a cancer-promoting role by inhibiting the expression of EYA1. This is consistent with decreased expression of EYA1 in gastric tumor samples, and EYA1 is inversely correlated with tumor size, lymphatic infiltration, and distant metastasis [37].

\section{Conclusion}

In conclusion, the results have shown that by targeting EYA1, miR-622 regulates breast cancer MCF-7 cell line proliferation, migration, and invasion, suggesting that miR-622 may become a potential target for breast cancer treatment. Reducing the production of miR-622 should be considered a promising strategy for targeted therapy of breast cancer.

\section{Abbreviations}

$\begin{array}{ll}\text { CCK-8: } & \text { Cell Counting Kit-8 } \\ \text { EMT: } & \text { Epithelial-mesenchymal transition } \\ \text { FBS: } & \text { Fetal bovine serum } \\ \text { HE: } & \text { Hematoxylin-eosin } \\ \text { miRNAs: } & \text { MicroRNAs } \\ \text { miR-NC: } & \text { miRNA negative control } \\ \text { P/S: } & \text { Penicillin-streptomycin } \\ \text { qRT-PCR: } & \text { Quantitative reverse transcriptase-polymerase } \\ & \text { chain reaction } \\ \text { RDGN: } & \text { Retinal determinant gene network } \\ \text { RIPA: } & \text { Radioimmunoprecipitation assay lysate } \\ \text { siRNA-NC: } & \text { siRNA negative control } \\ \text { si-EYA1: } & \text { EYA1 siRNA. }\end{array}$

\section{Data Availability}

The data used to support the findings of this study are available from the corresponding author upon request.

\section{Conflicts of Interest}

The authors declare that they have no conflicts of interest.

\section{Acknowledgments}

This work was supported by the Hunan Provincial Technology Innovation Guiding Plan-Clinical Medical Technology Innovation Guiding Project (2018SK51706).

\section{References}

[1] F. Bray, J. Ferlay, I. Soerjomataram, R. L. Siegel, L. A. Torre, and A. Jemal, "Global cancer statistics 2018: GLOBOCAN estimates of incidence and mortality worldwide for 36 cancers in 185 countries," CA: a Cancer Journal for Clinicians, vol. 68, no. 6, pp. 394-424, 2018.

[2] H. Sung, J. Ferlay, R. L. Siegel et al., "Global cancer statistics 2020: GLOBOCAN estimates of incidence and mortality worldwide for 36 cancers in 185 countries," CA: a Cancer Journal for Clinicians, vol. 71, no. 3, pp. 209-249, 2021.

[3] R. L. Siegel, K. D. Miller, and A. Jemal, "Cancer statistics, 2020," CA: a Cancer Journal for Clinicians, vol. 70, no. 1, pp. 7-30, 2020.

[4] R. L. Siegel, K. D. Miller, and A. Jemal, "Cancer statistics, 2018," CA: a Cancer Journal for Clinicians, vol. 68, no. 1, pp. 7-30, 2018.

[5] A. B. Hanker, D. R. Sudhan, and C. L. Arteaga, "Overcoming endocrine resistance in breast cancer," Cancer Cell, vol. 37, no. 4, pp. 496-513, 2020.

[6] J. Yang, A. Liu, I. He, and Y. Bai, "Bioinformatics analysis revealed novel $3^{\prime}$ UTR variants associated with intellectual disability," Genes, vol. 11, no. 9, p. 998, 2020.

[7] X. Pan, Z. Li, L. Zhao et al., "microRNA-572 functions as an oncogene and a potential biomarker for renal cell carcinoma prognosis," Oncology Reports, vol. 40, no. 5, pp. 3092-3101, 2018.

[8] X. Y. Shi, H. Wang, W. Wang, and Y. H. Gu, "MiR-98-5p regulates proliferation and metastasis of MCF-7 breast cancer cells by targeting Gab2," European Review for Medical and Pharmacological Sciences, vol. 24, no. 21, p. 10914, 2020.

[9] Y. K. Liang, H. Y. Lin, X. W. Dou et al., "MiR-221/222 promote epithelial-mesenchymal transition by targeting Notch3 in breast cancer cell lines," NPJ Breast Cancer, vol. 4, no. 1, p. 20, 2018.

[10] A. X. Liu, F. Yang, L. Huang, L. Y. Zhang, J. R. Zhang, and R. N. Zheng, "Long non-coding RNA tubulin alpha $4 \mathrm{~B}$ (TUBA4B) inhibited breast cancer proliferation and invasion by directly targeting miR-19," European Review for Medical and Pharmacological Sciences, vol. 23, no. 2, pp. 708-715, 2019.

[11] C. Han, Y. Fu, N. Zeng, J. Yin, and Q. Li, "LncRNA FAM83HAS1 promotes triple-negative breast cancer progression by regulating the miR-136-5p/metadherin axis," Aging, vol. 12, no. 4, pp. 3594-3616, 2020.

[12] S. Liu, L. Chen, H. Chen, K. Xu, X. Peng, and M. Zhang, "Circ_ 0119872 promotes uveal melanoma development by regulating the miR-622/G3BP1 axis and downstream signalling pathways," Journal of Experimental \& Clinical Cancer Research: CR, vol. 40, no. 1, p. 66, 2021. 
[13] Y. Zhang, Z. Li, and Z. Lan, "Retracted article: Silencing UNC5B antisense lncRNA 1 represses growth and metastasis of human Colon cancer cells via raising miR-622," Artificial Cells, Nanomedicine, and Biotechnology, vol. 48, no. 1, pp. 60-67, 2020.

[14] R. Wang, Q. Ma, L. Ji, Y. Yao, M. Ma, and Q. Wen, “miR-622 suppresses tumor formation by directly targeting VEGFA in papillary thyroid carcinoma," OncoTargets and therapy., vol. 11, pp. 1501-1509, 2018.

[15] F. M. Orlandella, R. M. Mariniello, P. Mirabelli et al., "miR-622 is a novel potential biomarker of breast carcinoma and impairs motility of breast cancer cells through targeting NUAK1 kinase," British Journal of Cancer, vol. 123, no. 3, pp. 426437, 2020.

[16] C. Liu, L. Min, J. Kuang, C. Zhu, X. Y. Qiu, and L. Zhu, "Bioinformatic identification of miR-622 key target genes and experimental validation of the miR-622-RNF8 axis in breast cancer," Frontiers in Oncology, vol. 9, p. 1114, 2019.

[17] Y. Okabe, T. Sano, and S. Nagata, "Regulation of the innate immune response by threonine-phosphatase of eyes absent," Nature, vol. 460, no. 7254, pp. 520-524, 2009.

[18] M. A. Blevins, C. G. Towers, A. N. Patrick, R. Zhao, and H. L. Ford, "The SIX1-EYA transcriptional complex as a therapeutic target in cancer," Expert Opinion on Therapeutic Targets, vol. 19, no. 2, pp. 213-225, 2015.

[19] D. Kong, A. Li, Y. Liu et al., "SIX1 activates STAT3 signaling to promote the proliferation of thyroid carcinoma via EYA1," Frontiers in oncology., vol. 9, p. 1450, 2019.

[20] J. Wu, B. Huang, H. B. He, W. Z. Lu, W. G. Wang, and H. Liu, "Two naturally derived small molecules disrupt the sineoculis homeobox homolog 1-eyes absent homolog 1 interaction to inhibit colorectal cancer cell growth," Chinese medical journal., vol. 134, no. 19, pp. 2340-2352, 2021.

[21] K. Wu, Z. Li, S. Cai et al., "EYA1 phosphatase function is essential to drive breast cancer cell proliferation through cyclin D1," Cancer Research, vol. 73, no. 14, pp. 4488-4499, 2013.

[22] H. Guan, Z. Dai, Y. Ma, Z. Wang, X. Liu, and X. Wang, "MicroRNA-101 inhibits cell proliferation and induces apoptosis by targeting EYA1 in breast cancer," International Journal of Molecular Medicine, vol. 37, no. 6, pp. 1643-1651, 2016.

[23] C. L. Chaffer and R. A. Weinberg, "A perspective on cancer cell metastasis," Science (New York, N.Y.), vol. 331, no. 6024, pp. 1559-1564, 2011.

[24] T. Shien and H. Iwata, "Adjuvant and neoadjuvant therapy for breast cancer," Japanese Journal of Clinical Oncology, vol. 50, no. 3, pp. 225-229, 2020.

[25] H. Wang, Z. Tan, H. Hu et al., "microRNA-21 promotes breast cancer proliferation and metastasis by targeting LZTFL1," BMC Cancer, vol. 19, no. 1, p. 738, 2019.

[26] B. Lin, C. Liu, E. Shi et al., "MiR-105-3p acts as an oncogene to promote the proliferation and metastasis of breast cancer cells by targeting GOLIM4," BMC Cancer, vol. 21, no. 1, p. 275 , 2021.

[27] A. Rodríguez-Martínez, D. de Miguel-Pérez, F. G. Ortega et al., "Exosomal miRNA profile as complementary tool in the diagnostic and prediction of treatment response in localized breast cancer under neoadjuvant chemotherapy," Breast Cancer Research: BCR, vol. 21, no. 1, p. 21, 2019.

[28] X. Y. Wang, M. H. Wu, F. Liu et al., "Differential miRNA expression and their target genes between NGX6-positive and negative colon cancer cells," Molecular and Cellular Biochemistry, vol. 345, no. 1-2, pp. 283-290, 2010.

[29] T. Li, X. Sun, and K. Xu, "The suppressing role of miR-622 in renal cell carcinoma progression by down-regulation of CCL18/MAPK signal pathway," Cell \& Bioscience, vol. 8, no. 1, p. 17, 2018.

[30] Y. F. Wu, Z. R. Li, Z. Q. Cheng, X. M. Yin, and J. S. Wu, "Decrease of miR-622 expression promoted the proliferation, migration and invasion of cholangiocarcinoma cells by targeting regulation of c-Myc," Biomedicine \& Pharmacotherapy $=$ Biomedecine \& Pharmacotherapie, vol. 96, pp. 7-13, 2017.

[31] C. Song, P. Lu, W. Shi et al., "MiR-622 functions as a tumor suppressor and directly targets E2F1 in human esophageal squamous cell carcinoma," Biomedicine \& Pharmacotherapy = Biomedecine \& Pharmacotherapie, vol. 83, pp. 843-849, 2016.

[32] Y. Wang, J. Sun, X. Wei et al., “Decrease of miR-622 expression suppresses migration and invasion by targeting regulation of DYRK2 in colorectal cancer cells," OncoTargets and Therapy, vol. Volume 10, pp. 1091-1100, 2017.

[33] Y. Fang, B. Sun, Z. Li, Z. Chen, and J. Xiang, "MiR-622 inhibited colorectal cancer occurrence and metastasis by suppressing K-Ras," Molecular Carcinogenesis, vol. 55, no. 9, pp. 1369-1377, 2016.

[34] J. Li, Y. Rodriguez, C. Cheng et al., "EYAl's conformation specificity in dephosphorylating phosphothreonine in Myc and its activity on Myc stabilization in breast cancer," Molecular and Cellular Biology, vol. 37, no. 1, 2017.

[35] J. Kim, C. She, M. Potez et al., "Phage display targeting identifies EYA1 as a regulator of glioblastoma stem cell maintenance and proliferation," Stem Cells, vol. 39, no. 7, pp. 853$865,2021$.

[36] D. Kong, W. Ma, D. Zhang et al., "EYA1 promotes cell migration and tumor metastasis in hepatocellular carcinoma," American Journal of Translational Research, vol. 11, no. 4, pp. 2328-2338, 2019.

[37] P. Nikpour, M. Emadi-Baygi, E. Emadi-Andani, and S. Rahmati, "EYAl expression in gastric carcinoma and its association with clinicopathological characteristics: a pilot study," Medical Oncology (Northwood, London, England), vol. 31, no. 5, p. 955, 2014. 